\title{
Microstructure of Thick Polycrystalline Silicon Films for MEMS Application
}

\author{
H.W. Zhou* and B.G. Kharas**, P.I. Gouma* \\ * Dept. of Materials Science and Engineering, State University of New York at Stony Brook, NY \\ 11794 \\ ** Standard MEMS, Inc., 35 Marcus Boulevard, Hauppauge, NY 11788
}

Thick polysilicon layers $(\geqslant 0.5 \mu \mathrm{m})$ become increasingly attractive for use as MEMS structure materials ${ }^{[1-3]}$. Increased film thickness improves mechanical rigidity in applications such as pressure sensor, provides increased surface area in comb-drive actuators, or increased mass in accelerometer applications. Thicker films however, can experience greater variability in morphology and in surface roughness due to longer deposition times.

$3.0 \mu \mathrm{m}$ thick polysilicon films were deposited on silicon wafers by the lower pressure chemical vapor deposition (LPCVD) process. The films were grown by the thermal decomposition of silane in a horizontal hot walled LPCVD furnace at a temperature of $590^{\circ} \mathrm{C}$. The problem addressed is the observed heterogeneity in the film quality that is associated with the position of the wafer in the furnace during film deposition. The microstructural characteristics of films produced in the same batch but differed in the location of the furnace were examined by means of high resolution scanning electron microscopy and electron diffraction analysis in a transmission electron microscope (TEM). Tripod polishing was used to prepare cross-sectional samples of the polysilicon films. Mechanical and electrical property measurements were also conducted to evaluate the effect of the heterogeneity in the films' performance.

Asperities with diameters from 100 micron to 800 micron were observed on the surface of the polysilicon layers. TEM studies showed the co-existence of ellipsoidal fine grains and some discrete long columnar crystallites containing microtwins (fine laths) in the materials microstructure. The asperities on the surface are found to be the caps of the columnar grains that grow continuously out of the flat surface of the ellipsoidal grains. The density of these asperities is much more larger for the films deposited at the end of the furnace than those at the entrance.

The structure evolution of the thick polysilicon layers was formed by the non-uniform conditions in the furnace that affected the nucleation and growth process, in particular the variation of silane gas pressure in the furnace ${ }^{[4]}$. At the entrance of the gas flow, the silicon crystal nucleation rate on the growing planes is high because of a higher silane pressure. Thus, a structure with more volume fraction of ellipsoidal fine grains is likely to be formed. While at the end of the furnace, the partial pressure of silane is low which results in a lower nucleation rate. So those favourably oriented nuclei whose fast growing direction is the same as the film growing direction are likely to form the observed long columnar crystallites, resulting in a microstructure with co-existence of both columnar and ellipsoidal grains.

References

[1] H. Kahn et al., Philosophical Magazine A. 82 (2002) 137.

[2] S. Greek et al. Thin Solid Films. 292 (1997) 247.

[3] L. Haji et al. J. Appl. Phys. 75 (1994) 3944. 
[4] B.G. Kharas et al. Proc. of the Mat. Res. Soc. 648 (2000) P6.48

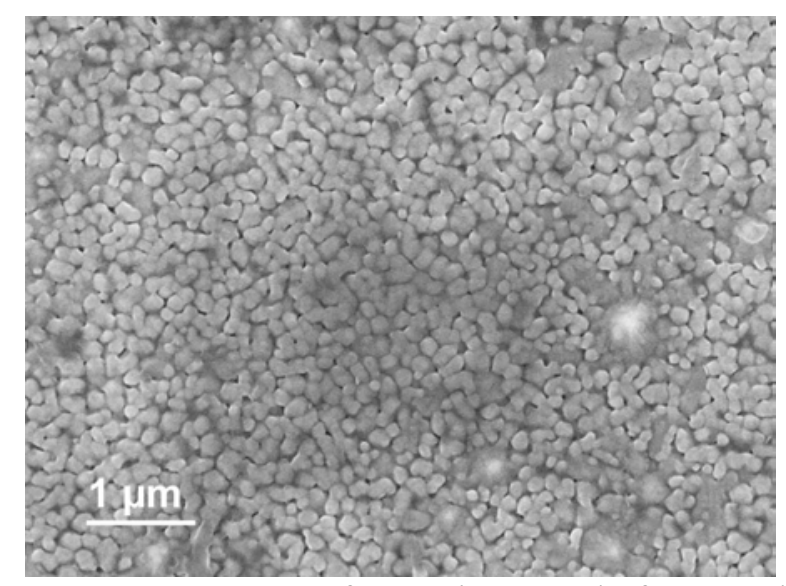

FIG. 1. SEM surface micrograph from a thick poly-Si film deposited by LPCVD at $590^{\circ} \mathrm{C}$ and wafer position close to the entrance of the gas flow

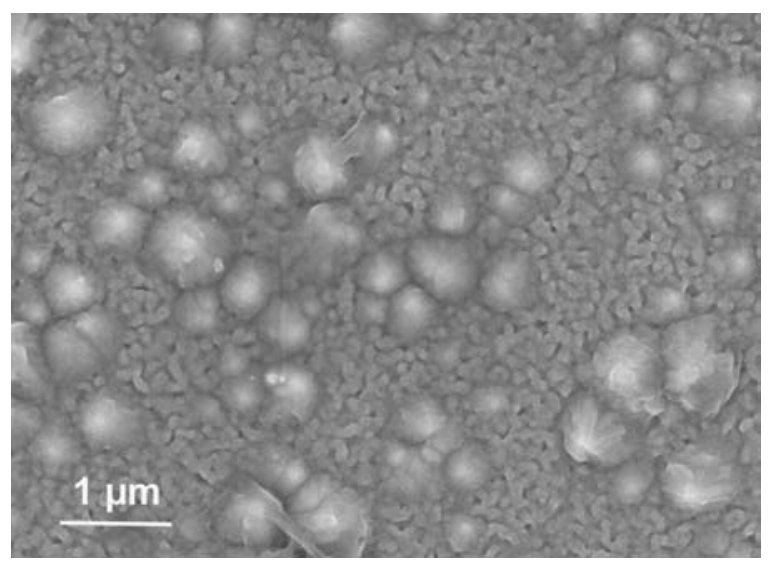

FIG. 2. SEM surface micrograph from a thick poly-Si film deposited by LPCVD at $590^{\circ} \mathrm{C}$ and wafer position far from the entrance of the gas flow

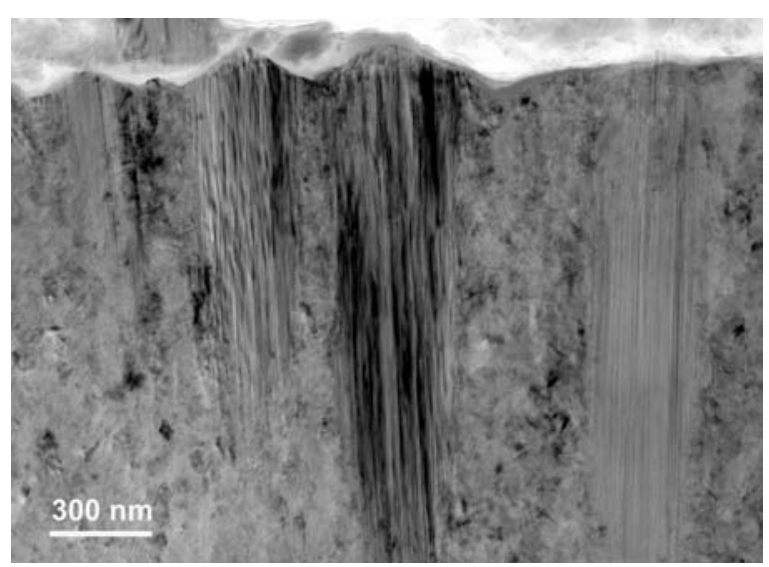

FIG. 3. TEM cross-sectional micrograph from a thick poly-Si film deposited by LPCVD at $590^{\circ} \mathrm{C}$ and wafer position far from the entrance of the gas flow. 